\title{
2.5D discrete-dual-porosity model for simulating geoelectrical experiments in fractured rock
}

February 20, 2017

\author{
Victor Caballero Sanz ${ }^{\dagger, a}$, Delphine Roubinet ${ }^{\dagger, a, b, *}$, Serdar Demirel $^{a}$, and James Irving ${ }^{a}$ \\ ${ }^{a}$ Applied and Environmental Geophysics Group, Institute of Earth Sciences, University of Lausanne, \\ Switzerland \\ ${ }^{b}$ Now at Geosciences Montpellier, CNRS UMR 5243, University of Montpellier, France \\ $\dagger$ : These authors contributed equally to this research. \\ *: Corresponding author (delphine.roubinet@umontpellier.fr)
}

Draft for submission to Geophysical Journal International

2 Abbreviated title: 2.5D geoelectrical modeling in fractured rock

\section{Abstract}

4 Previous work has demonstrated that geoelectrical measurements, acquired either along the Earth's 5 surface or in boreholes, can be sensitive to the presence of fractures. However, a lack of numerical 6 approaches that are well suited to modeling electric current flow in fractured media prevents us from 7 systematically exploring the links between geoelectrical measurements and fractured rock properties. To address this issue, we present a highly computationally efficient methodology for the numerical simulation of geoelectrical data in 2.5 dimensions in complex fractured domains. Our approach is based upon a discrete-dual-porosity formulation, whereby the fractures and rock matrix are treated separately and coupled through the exchange of electric current between them. We first validate our methodology against standard analytical and finite-element solutions. Subsequent use of the approach to simulate geoelectrical data for a variety of different fracture configurations demonstrates the sensitivity of these data to important parameters such as the fracture density, depth, and orientation.

15

Keywords: electrical properties, electrical resistivity tomography (ERT), fracture and flow, numerical modeling, numerical solutions, Fourier analysis 


\section{Introduction}

The study of fractured rocks is extremely important for many applications including aquifer assessment and remediation, geothermal and hydrothermal resource exploitation, hydrocarbon extraction, and the long-term storage of toxic waste (e.g., Carneiro, 2009; Dershowitz \& Miller, 1995; Gautam \& Mohanty, 2004; Rotter et al., 2008). As a result, numerous studies have been devoted to detecting these highlyconductive structures, evaluating their geometrical and physical properties, and determining how they are distributed and connected (e.g., Berkowitz, 2002; Bonnet et al., 2001; Neuman, 2005). In particular, the use of geophysical methods, notably seismic, ground-penetrating radar, electrical resistivity, induced polarization, self-potential, and electromagnetic methods, has been extensively investigated (e.g., Dorn et al., 2011; Lofi et al., 2012; Robinson et al., 2013; Wishart et al., 2008). Here, we focus on the electrical resistivity method because (i) it has been shown that field geoelectrical measurements are impacted by the presence of fractures (e.g., Boadu et al., 2005; Busby, 2000; Lane et al., 1995); (ii) the possibility exists for important hydraulic information to be obtained from geoelectrical data because fractures represent preferential pathways for both fluid and electric current flow (e.g., Brown, 1989; Ritzi \& Andolsek, 1992; Nguyen et al., 2016; Kirkby et al., 2016); and (iii) geoelectrical measurements can be acquired in a straightforward manner along the Earth's surface and from boreholes over a wide range of spatial scales.

In order to understand in detail the impact of fractures on geoelectrical data with the overall goal of exploring how such data might be eventually utilized to identify subsurface fractures and estimate their properties, accurate numerical models for electric current flow in fractured media are required. When the considered subsurface domain can be treated as a representative elementary volume (REV) at the scale of the geoelectrical measurements, development of such models is relatively straightforward because the fractured medium can be defined in terms of an electrical conductivity tensor at each subsurface location. In other words, in such cases, the fracture network will be dense enough with respect to the measurement scale to be effectively modeled as an anisotropic continuum (e.g., Herwanger et al., 2004a,b; Greenhalgh et al., 2009a,b; Shen et al., 2009; Li \& Spitzer, 2005). In the common case where the REV assumption is not appropriate, however, the fractures must be explicitly represented. This poses severe problems for standard numerical approaches such as finite-element or finite-volume methods because they rapidly become computationally prohibitive as the number of fractures increases. Indeed, only a small number of fractures can be considered with such standard approaches because each fracture, whose aperture is typically many orders of magnitude smaller than the size of the domain being investigated, must be discretized (e.g., Robinson et al., 2013). 
In this paper, we address the above challenge and present a highly computationally efficient methodology for numerically simulating geoelectrical experiments in heterogeneous and complex fractured domains. Our approach builds on the recently developed 2D discrete-dual-porosity (DDP) model for electric current flow in fractured media developed by Roubinet \& Irving (2014), whereby fractures are explicitly represented using a semi-analytical formulation that takes into account the exchange of electric current flow between the fractures and surrounding matrix. However, we importantly redevelop this formulation for the $2.5 \mathrm{D}$ case, commonly considered in geoelectrical imaging, in order to accurately simulate current flow between point electrodes. The mathematical formulation of our new numerical method, including the general problem formulation in $2.5 \mathrm{D}$ and the corresponding DDP modeling approach, is presented in Section 2. In Section 3, we validate our approach for both unfractured and fractured porous domains considering, in the latter case, both simple and complex fracture networks. Finally, we use our model in Section 4 to simulate ERT experiments in fractured porous domains composed of idealized and realistic fracture networks.

\section{Modeling approach}

\subsection{General problem formulation}

Consider a three-dimensional domain having electrical conductivity $\sigma(x, y, z)[\mathrm{S} / \mathrm{m}]$ in which an electric current $I[\mathrm{~A}]$ is injected at position $\left(x_{0}, y_{0}, z_{0}\right)$. Under steady-state conditions, the current flow in this domain is governed by the following charge-conservation equation at the point scale:

$$
-\nabla \cdot[\sigma(x, y, z) \vec{\nabla} \phi(x, y, z)]=I \delta\left(x-x_{0}\right) \delta\left(y-y_{0}\right) \delta\left(z-z_{0}\right)
$$

where $\phi(x, y, z)[\mathrm{V}]$ is the electric potential and $\delta().\left[\mathrm{m}^{-1}\right]$ is the Dirac delta function. Assuming that (i) the electrical conductivity $\sigma$ is constant in the $y$-direction (i.e., $\sigma(x, y, z)=\sigma(x, z)$ and $\partial_{y} \sigma=0$ );

(ii) the considered problem is symmetric in the $y$-direction (i.e., $\phi(x, y, z)=\phi(x,-y, z)$ ); and (iii) the current injection lies in the $y=0$ plane (i.e., $y_{0}=0$ ), equation (1) can be expressed in the Fourier domain as follows (e.g., Dey \& Morrison, 1979)

$$
-\nabla \cdot[\sigma(x, z) \vec{\nabla} \bar{\phi}(x, \omega, z)]+\omega^{2} \sigma(x, z) \bar{\phi}(x, \omega, z)=\frac{I}{2} \delta\left(x-x_{0}\right) \delta\left(z-z_{0}\right)
$$


where $\bar{\phi}(x, \omega, z)$ is the Fourier-cosine transform of $\phi(x, y, z)$ and $\omega$ is the wavenumber corresponding to the $y$-coordinate. The distributions of potential $\phi$ and $\bar{\phi}$ are related through (e.g., Bateman, 1954):

$$
\bar{\phi}(x, \omega, z)=\int_{0}^{\infty} \phi(x, y, z) \cos (\omega y) \mathrm{d} y
$$

$$
\phi(x, y, z)=\frac{2}{\pi} \int_{0}^{\infty} \bar{\phi}(x, \omega, z) \cos (\omega y) \mathrm{d} \omega
$$

Equation (2) corresponds to the 2.5D formulation of equation (1), whereby the $3 \mathrm{D}$ problem is decomposed into series of $2 \mathrm{D}$ problems in the Fourier domain. That is, under the assumptions stated above, the $3 \mathrm{D}$ electric potential $\phi(x, y, z)$ in equation (1) can be determined by solving equation (2) in the Fourier domain for several values of $\omega$, and then inverting the resulting $\bar{\phi}(x, \omega, z)$ using the inverse Fourier-cosine transform (3b). Appendix A describes how this inverse Fourier-cosine transform is implemented and how the choice of wavenumber values is optimized in our work. The DDP formulation used to solve equation (2) for heterogeneous and complex fractured domains is described next.

\subsection{Discrete-dual-porosity approach}

To develop a DDP formulation of the electric current flow problem (2) in the Fourier domain, we build upon the 2D formulation presented by Roubinet \& Irving (2014). In this formulation, the fractures and matrix are treated separately and coupled through the exchange of electric current between them. The fractures and matrix are discretized into fracture segments and matrix blocks having constant properties, respectively, and a linear system is created where the unknowns are the electrical potentials at the fracture intersections and extremities, as well as in the matrix blocks. Below, we derive the corresponding 2.5D equations at the fracture-segment (Section 2.2.1), fracture-network (Section 2.2.2) and matrix-block (Section 2.2.3) scales. In doing this, it is assumed that fractures extend infinitely perpendicular to the $2 \mathrm{D}$ modeling plane being considered. Note that our presentation contains only the key differences between this 2.5D DDP formulation and the work of Roubinet \& Irving (2014). For full information on the representation and discretization methods used to model the geological structures as well as on the solution of the linear system, please see their paper. 


\subsubsection{Electric potential along a fracture segment}

For each 1D fracture-segment $k$ having constant aperture $b_{f}^{k}$ and electrical conductivity $\sigma_{f}^{k}$, consider the charge conservation equation (2) in the Fourier domain

$$
-\sigma_{f}^{k} \partial_{x_{f}^{k}}^{2} \bar{\phi}_{f}^{k}+\omega^{2} \sigma_{f}^{k} \bar{\phi}_{f}^{k}=-\bar{Q}_{f m}^{k}
$$

where $\bar{\phi}_{f}^{k}=\bar{\phi}_{f}^{k}\left(x_{f}^{k}\right)$ is the Fourier-cosine transform of the electric potential averaged over the fracture aperture, $x_{f}^{k}$ denotes the spatial variable along the fracture segment, and $\bar{Q}_{f m}^{k}$ is the Fourier-cosine transform of the source term related to the exchange of electric current between the fracture segment and the surrounding matrix. Considering that this fracture segment is located within matrix block $\left(I_{k}, J_{k}\right)$, where $\bar{\phi}_{m}^{I_{k}, J_{k}}$ is the Fourier-cosine transform of the electric potential in this block, $\bar{Q}_{f m}^{k}$ can be expressed as

$$
\bar{Q}_{f m}^{k}=-\alpha_{f m}^{I_{k}, J_{k}}\left(\bar{\phi}_{m}^{I_{k}, J_{k}}-\bar{\phi}_{f}^{k}\right)
$$

Here, $\alpha_{f m}^{I_{k}, J_{k}}$ represents the fracture-matrix exchange coefficient, defined as $\alpha_{f m}^{I_{k}, J_{k}}=\sigma_{m}^{I_{k}, J_{k}} / d_{f m}^{I_{k}, J_{k}}$, where $\sigma_{m}^{I_{k}, J_{k}}$ is the matrix electrical conductivity of block $\left(I_{k}, J_{k}\right)$ and $d_{f m}^{I_{k}, J_{k}}$ is the average normal distance between the fractures in that block and each point in the block (Roubinet \& Irving, 2014; Roubinet et al., 2016).

We consider Fourier-domain Dirichlet boundary conditions $\bar{\varphi}_{f}^{i_{k}}$ and $\bar{\varphi}_{f}^{j_{k}}$ at the extremities of each fracture segment $x_{k}=0$ and $x_{k}=L_{k}$, respectively. Solving analytically equation (4) with these conditions leads to the following expression for $\bar{\phi}_{f}^{k}$ :

$$
\bar{\phi}_{f}^{k}\left(x_{k}, \omega\right)=\beta_{w}\left(x_{k}\right) \bar{\varphi}_{f}^{i_{k}}+\frac{\gamma_{w}\left(x_{k}\right)}{\gamma_{w}\left(L_{k}\right)} \bar{\varphi}_{f}^{j_{k}}+\frac{\Gamma_{I_{k}, J_{k}}^{k}}{\Gamma_{I_{k}, J_{k}}^{k}+\omega^{2}}\left[1-\beta_{w}\left(x_{k}\right)-\frac{\gamma_{w}\left(x_{k}\right)}{\gamma_{w}\left(L_{k}\right)}\right] \bar{\phi}_{m}^{I_{k}, J_{k}}
$$

with

$$
\begin{aligned}
& \Gamma_{I_{k}, J_{k}}^{k} \equiv \alpha_{f m}^{I_{k}, J_{k}} /\left(b_{f}^{k} \sigma_{f}^{k}\right) \\
& \beta_{w}\left(x_{k}\right)=\exp \left(x_{k} \sqrt{\Gamma_{I_{k}, J_{k}}^{k}+\omega^{2}}\right)-\frac{\gamma_{w}\left(x_{k}\right)}{\gamma_{w}\left(L_{k}\right)} \exp \left(L_{k} \sqrt{\Gamma_{I_{k}, J_{k}}^{k}+\omega^{2}}\right) \\
& \gamma_{w}\left(x_{k}\right)=\exp \left(-x_{k} \sqrt{\Gamma_{I_{k}, J_{k}}^{k}+\omega^{2}}\right)-\exp \left(x_{k} \sqrt{\Gamma_{I_{k}, J_{k}}^{k}+\omega^{2}}\right)
\end{aligned}
$$




\subsubsection{Modified DFN approach for the fracture network}

At the fracture-network scale, charge conservation at each fracture-intersection node is enforced by integrating equation (2) over the intersection. For simplification, we consider that every node $i$ is shared by $N_{i}$ fracture segments having the same aperture $b_{f}^{i}$ and conductivity $\sigma_{f}^{i}$, and that the surface of this intersection can be approximated by $b_{f}^{i} \times b_{f}^{i}$. Applying Gauss's Divergence theorem leads to

$$
b_{f}^{i} \omega^{2} \sigma_{f}^{i} \bar{\phi}_{f \mid x_{f}^{k}=0}^{i}-\sigma_{f}^{i} \sum_{k=1}^{N_{i}} \partial_{x_{f}^{k}} \bar{\phi}_{f \mid x_{f}^{k}=0}^{k}=0 .
$$

Using expression (6), equation (8) can be rewritten as:

$$
b_{f}^{i} \omega^{2} \sigma_{f}^{i} \bar{\varphi}_{f}^{i_{k}}-\sigma_{f}^{i} \sum_{k=1}^{N_{i}}\left(A_{i_{k}} \bar{\varphi}_{f}^{i_{k}}+A_{j_{k}} \bar{\varphi}_{f}^{j_{k}}+A_{I_{k}, J_{k}} \bar{\phi}_{m}^{I_{k}, J_{k}}\right)=0
$$

where the terms $A_{i_{k}}, A_{j_{k}}$, and $A_{I_{k}, J_{k}}$ are defined as

$$
\begin{aligned}
& A_{i_{k}}=\zeta_{w}\left(x_{k}\right) \sqrt{\Gamma_{I_{k}, J_{k}}^{k}+\omega^{2}} \\
& A_{j_{k}}=-\frac{\lambda_{w}\left(x_{k}\right)}{\gamma_{w}\left(L_{k}\right)} \sqrt{\Gamma_{I_{k}, J_{k}}^{k}+\omega^{2}} \\
& A_{I_{k}, J_{k}}=-\frac{\Gamma_{I_{k}, J_{k}}^{k}}{\Gamma_{I_{k}, J_{k}}^{k}+\omega^{2}}\left(A_{i_{k}}+A_{j_{k}}\right)
\end{aligned}
$$

with

$$
\begin{aligned}
& \zeta_{w}\left(x_{k}\right)=\exp \left(x_{k} \sqrt{\Gamma_{I_{k}, J_{k}}^{k}+\omega^{2}}\right)+\frac{\lambda_{w}\left(x_{k}\right)}{\gamma_{w}\left(L_{k}\right)} \exp \left(L_{k} \sqrt{\Gamma_{I_{k}, J_{k}}^{k}+\omega^{2}}\right) \\
& \lambda_{w}\left(x_{k}\right)=\exp \left(x_{k} \sqrt{\Gamma_{I_{k}, J_{k}}^{k}+\omega^{2}}\right)+\exp \left(-x_{k} \sqrt{\Gamma_{I_{k}, J_{k}}^{k}+\omega^{2}}\right) .
\end{aligned}
$$

\subsubsection{Modified finite-volume approach in the matrix}

Finally, in the matrix, charge conservation is enforced in the Fourier domain by integrating equation (2) over each matrix block $(I, J)$ of volume $V_{I, J}$. This leads to

$$
-\int_{V_{I, J}} \nabla \cdot\left(\sigma_{m} \vec{\nabla} \bar{\phi}_{m}^{I, J}\right) \mathrm{d} V+\int_{V_{I, J}} \omega^{2} \sigma_{m} \bar{\phi}_{m}^{I, J} \mathrm{~d} V=\int_{V_{I, J}} \bar{Q}_{f m}^{k} \mathrm{~d} V
$$


Using Gauss' Divergence Theorem, the left-hand side of equation (12), which we denote as $M_{I, J}$, can be discretized as

$$
M_{I, J}=C_{I, J} \bar{\phi}_{m}^{I, J}+C_{I, J}^{W} \bar{\phi}_{m}^{I-1, J}+C_{I, J}^{E} \bar{\phi}_{m}^{I+1, J}+C_{I, J}^{S} \bar{\phi}_{m}^{I, J-1}+C_{I, J}^{N} \bar{\phi}_{m}^{I, J+1},
$$

where

$$
\begin{aligned}
C_{I, J}^{W} & =-\frac{\Delta z}{\Delta x} \mathcal{H}_{(I-1, J),(I, J)} \\
C_{I, J}^{E} & =-\frac{\Delta z}{\Delta x} \mathcal{H}_{(I+1, J),(I, J)} \\
C_{I, J}^{S} & =-\frac{\Delta x}{\Delta z} \mathcal{H}_{(I, J-1),(I, J)} \\
C_{I, J}^{N} & =-\frac{\Delta x}{\Delta z} \mathcal{H}_{(I, J+1),(I, J)} \\
C_{I, J} & =\omega^{2} \sigma_{m}^{I, J} \Delta x \Delta z-C_{I, J}^{W}-C_{I, J}^{E}-C_{I, J}^{S}-C_{I, J}^{N}
\end{aligned}
$$

with $\mathcal{H}_{(K, L),(I, J)}$ the harmonic mean of the electrical conductivity in matrix blocks $(K, L)$ and $(I, J)$, i.e., $\mathcal{H}_{(K, L),(I, J)}=2 /\left(1 / \sigma_{m}^{K, L}+1 / \sigma_{m}^{I, J}\right)$.

The right-hand side of equation (12) can be expressed as

$$
\int_{V_{I, J}} \bar{Q}_{f m}^{k} \mathrm{~d} V=\sum_{k=1}^{N_{I, J}^{f}} \int_{0}^{L_{k}} \bar{Q}_{f m}^{k} \mathrm{~d} V
$$

where $N_{I, J}^{f}$ is the number of fractures contained in the matrix block volume $V_{I, J}$. Using expression (5) for the source term $\bar{Q}_{f m}^{k}$ leads to

$$
\int_{V_{I, J}} \bar{Q}_{f m}^{k} \mathrm{~d} V=-\alpha_{f m}^{I, J} \bar{\phi}_{m}^{I, J} \sum_{k=1}^{N_{I, J}^{f}} L_{k}+\alpha_{f m}^{I, J} \sum_{k=1}^{N_{I, J}^{f}} \bar{\Phi}_{f}^{k},
$$

where $\bar{\Phi}_{f}^{k}$ is the integrated value of $\bar{\phi}_{f}^{k}$ along fracture segment $k$, i.e., $\bar{\Phi}_{f}^{k}=\int_{0}^{L_{k}} \bar{\phi}_{f}^{k} \mathrm{~d} x_{k}$, and $\left(I_{k}, J_{k}\right)=$ $(I, J)$ for $k=1, \ldots, N_{I, J}^{f}$. Integrating expression (6) for $\bar{\phi}_{f}^{k}$, we obtain the following definition for $\bar{\Phi}_{f}^{k}$ :

$$
\bar{\Phi}_{f}^{k}=D_{i_{k}} \bar{\varphi}_{f}^{i_{k}}+D_{j_{k}} \bar{\varphi}_{f}^{j_{k}}+D_{I, J} \bar{\phi}_{m}^{I, J},
$$


where the coefficients $D_{i_{k}}, D_{j_{k}}$, and $D_{I, J}$ are defined as

$$
\begin{aligned}
D_{i_{k}} & =\frac{\zeta_{w}\left(L_{k}\right)-1}{\sqrt{\Gamma_{I_{k}, J_{k}}^{k}+\omega^{2}}}-\frac{2 \exp \left(\sqrt{\Gamma_{I_{k}, J_{k}}^{k}+\omega^{2}} L_{k}\right)}{\gamma_{w}\left(L_{k}\right) \sqrt{\Gamma_{I_{k}, J_{k}}^{k}+\omega^{2}}} \\
D_{j_{k}} & =\frac{2-\lambda_{w}\left(L_{k}\right)}{\gamma_{w}\left(L_{k}\right) \sqrt{\Gamma_{I_{k}, J_{k}}^{k}+\omega^{2}}} \\
D_{I, J} & =\frac{\Gamma_{I, J}^{k}}{\Gamma_{I, J}^{k}+\omega^{2}}\left(L_{k}-D_{j_{k}}-D_{i_{k}}\right) .
\end{aligned}
$$

Finally, the discretized expression of equation (12) is given by

$$
\begin{aligned}
& {\left[C_{I, J}+\alpha_{f m}^{I, J} \sum_{k=1}^{N_{I, J}^{f}}\left(L_{k}-D_{I, J}\right)\right] \bar{\phi}_{m}^{I, J}+C_{I, J}^{W} \bar{\phi}_{m}^{I-1, J}+C_{I, J}^{E} \bar{\phi}_{m}^{I+1, J}+C_{I, J}^{S} \bar{\phi}_{m}^{1, J-1}+C_{I, J}^{N} \bar{\phi}_{m}^{I, J+1}} \\
& -\alpha_{f m}^{I, J} \sum_{k=1}^{N_{I, J}^{f}}\left(D_{i_{k}} \bar{\varphi}_{f}^{i_{k}}+D_{j_{k}} \bar{\varphi}_{f}^{j_{k}}\right)=0 .
\end{aligned}
$$

\section{$3 \quad$ Validation}

We now validate our 2.5D modeling approach for unfractured (Section 3.1) and fractured (Section 3.2) porous domains considering a variety of different boundary conditions. We begin with simple configurations for which known analytical solutions exist (Sections 3.1 and 3.2.1). We then validate our approach for more complex configurations involving multiple fractures using a standard finite-element approach as a reference solution (Section 3.2.2).

\subsection{Unfractured porous domains}

Validating on unfractured porous domains enables us to verify the modified finite-volume formulation presented in Section 2.2.3. Here we consider the homogeneous and two-layer configurations presented in Figures 1a and $\mathrm{b}$, respectively. In these square domains of side length $L=30 \mathrm{~m}$, the electrical conductivities $\sigma_{1}$ and $\sigma_{2}$ are equal to $10^{-3}$ and $10^{-1} \mathrm{~S} / \mathrm{m}$, respectively, and the interface between the layers in Figure 1b is located at a depth of $z^{*}=1.5 \mathrm{~m}$. Zero electrical conductivity is assumed above each domain. In order to simulate an electrical resistivity experiment, surface point-source injections of electric current $I$ and $-I$ are considered $10 \mathrm{~m}$ apart at $x=10 \mathrm{~m}$ and $x=20 \mathrm{~m}$, respectively, with $I=1 \mathrm{~A}$.

As reference solutions, we consider the 3D analytical expressions for the electric potential corre- 


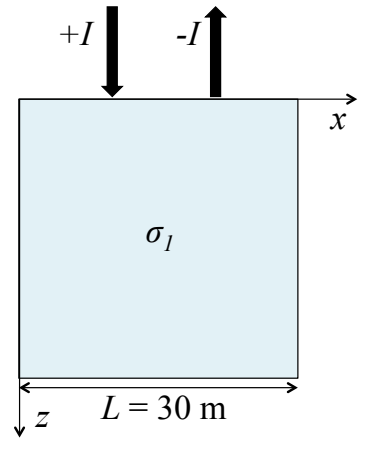

(a)

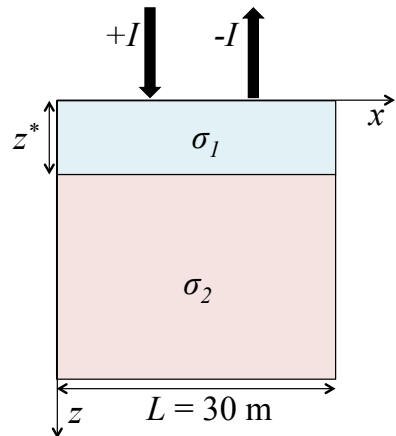

(b)

Figure 1: Configurations used to validate our modeling approach for unfractured porous media: (a) homogeneous domain and (b) two-layer domain.

sponding to Figures 1a and b, which we denote by $\phi_{3 D}^{r e f 1}$ and $\phi_{3 D}^{r e f 2}$, respectively. These analytical solutions assume that the considered domains extend infinitely into the subsurface and are given by (e.g., Telford et al., 1990):

$$
\begin{gathered}
\phi_{3 D}^{r e f 1}=\frac{I}{2 \pi \sigma_{1}}\left(\frac{1}{r_{1}}-\frac{1}{r_{2}}\right) \\
\phi_{3 D}^{r e f 2}=\frac{I}{2 \pi \sigma_{1}}\left\{\frac{1}{r_{1}}\left[1+2 \sum_{m=1}^{\infty} \frac{k^{m}}{\sqrt{1+\left(2 m z^{*} / r_{1}\right)^{2}}}\right]-\frac{1}{r_{2}}\left[1+2 \sum_{m=1}^{\infty} \frac{k^{m}}{\sqrt{1+\left(2 m z^{*} / r_{2}\right)^{2}}}\right]\right\},
\end{gathered}
$$

where $r_{1}$ and $r_{2}$ are the distances to the locations of the point-source injections $I$ and $-I$, respectively, and $k=\left(\sigma_{1}-\sigma_{2}\right) /\left(\sigma_{1}+\sigma_{2}\right)$. Considering that these injections are located at positions $\left(x_{1}, y_{1}, z_{1}\right)$ and $\left(x_{2}, y_{2}, z_{2}\right)$, with $y_{1}=y_{2}=0$ and $z_{1}=z_{2}=0$, the Fourier-cosine transform of (20) leads to the following 2.5D equations (e.g., Bateman, 1954):

$$
\bar{\phi}_{2.5 D}^{r e f 1}=\frac{I}{2 \pi \sigma_{1}}\left[\mathcal{K}_{0}\left(\omega \sqrt{k_{1}}\right)-\mathcal{K}_{0}\left(\omega \sqrt{k_{2}}\right)\right]
$$

where $\mathcal{K}_{0}(\cdot)$ is the modified Bessel function of the second kind of order $0, k_{1}=\left(x-x_{1}\right)^{2}+z^{2}$, and 
$k_{2}=\left(x-x_{2}\right)^{2}+z^{2}$.

As has been done in previous studies (e.g., Pidlisecky \& Knight, 2008), the domains in Figure 1 were discretized into regular cells when calculating both the analytical and numerical solutions, and the electric potential was determined at the center of each cell. This was done in order to (i) avoid the infinite values of the electric potential at the locations of the point-source injections; and (ii) facilitate the comparison between the analytical and numerical solutions since the electric potential distribution is evaluated at exactly the same positions in both cases. We considered 101 cells in each direction and we approximated the infinite sums in (20b) and (21b) using 100 terms. The discrete inverse Fourier-cosine transform (Appendix A) was used to invert the results obtained from the Fourier-domain analytical solution (21) and from our DDP modeling approach. For the homogeneous configuration (Figure 1a), we used our numerical approach exactly as presented in Section 2.2, whereas for the two-layer configuration (Figure 1b) the singularity removal technique presented in Appendix B was employed in order to improve the accuracy of the solution. This technique, as with all singularity removal methods, can only be applied to heterogeneous domains because it is based on the difference in potential between the considered heterogeneous configuration and its equivalent homogeneous configuration. For our DDP formulation, we considered an insulating boundary condition along the top of the studied domains, and the mixed boundary conditions described in Appendix $\mathrm{C}$ along the other borders. The final results are obtained by summing the distributions of electric potential determined separately for the point-source injections $I$ and $-I$.

Figure 2 shows the absolute value of the electric potential along the Earth's surface, $\phi_{s}$, for the configurations presented in Figure 1, computed using the 3D analytical solutions (20), the 2.5D analytical solutions (21), and our numerical approach. For both the homogeneous and two-layer configurations, we observe an excellent overall agreement between the analytical solutions and our numerical approach, which confirms the validation of the approach for unfractured porous domains. The only exception is near the location of the current electrodes at $x_{1}=10 \mathrm{~m}$ and $x_{2}=20 \mathrm{~m}$, where discrepancies between all solutions can be seen to exist because of the well-known singularity problem present at these locations (e.g., Pidlisecky \& Knight, 2008). By using the singularity removal technique presented in Appendix B, the differences between the 2.5D analytical solution and our numerical solution are reduced at these locations for the two-layer case. Note that simulations were also carried out using $z^{*}=9 \mathrm{~m}$ and $z^{*}=18 \mathrm{~m}$ for the two-layer configuration in Figure 1b, and showed excellent agreement between the 2.5D analytical solution and our numerical approach with the use of the removal singularity technique (results not shown). 


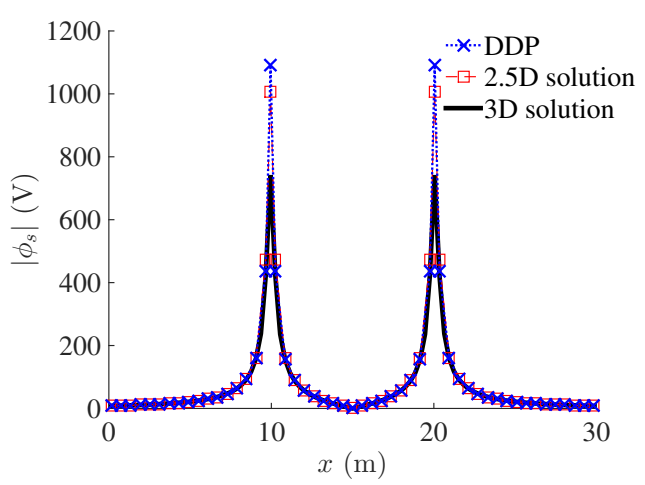

(a)

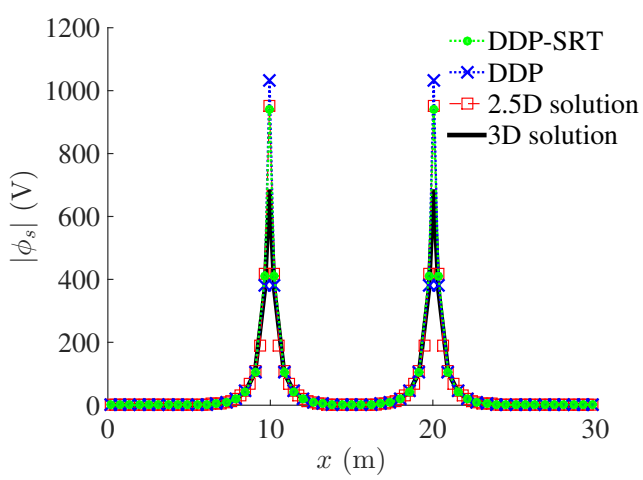

(b)

Figure 2: Absolute value of the electric potential at the Earth's surface, $\phi_{s}$ (in V), corresponding to the (a) homogeneous and (b) two-layer configurations presented in Figures 1a and b, respectively. Results were computed using our numerical approach, the 2.5D analytical solutions (21), and the 3D analytical solutions (20). In (b), we also show the results obtained using our numerical approach combined with a singularity removal technique (SRT).

\section{$3.2 \quad$ Fractured porous domains}

To validate our numerical modeling approach for fractured porous domains, we consider first a simple configuration involving a single horizontal fracture (Section 3.2.1). Then, we perform validations on three more complex configurations involving multiple fractures (Section 3.2.2). Standard analytical and finite-element solutions are used as reference solutions in the former and latter cases, respectively.

\subsubsection{Single horizontal fracture}

Consider a single horizontal fracture located at depth $z^{*}$ having aperture $b_{f}$ and electrical conductivity $\sigma_{f}$, and embedded in a matrix of electrical conductivity $\sigma_{m}$. We assume Dirichlet boundary conditions for the electric potential on the left and right sides of the domain equal to $1 \mathrm{~V}$ and $0 \mathrm{~V}$, respectively, and insulating boundary conditions on the top and bottom. These boundary conditions are widely 
employed in hydraulic and electrical conductivity modeling studies (e.g., Long et al., 1982; Roubinet et al., 2010; Roubinet \& Irving, 2014), and lead to the following analytical expression for the electric potential $\bar{\phi}_{S C}$ in the Fourier domain:

$$
\bar{\phi}_{S C}=\frac{\sin (\omega L)}{\omega\left(1-e^{2 \omega L}\right)}\left[e^{\omega x}-e^{-\omega(x-2 L)}\right],
$$

where $L$ is the length of the domain in the $x$-direction and $\omega$ is the wavenumber associated with the Fourier-cosine transform defined in (3a). Note that equation (22) has no dependence on the depth of the fracture $z^{*}$ and on the depth coordinate $z$, nor does it depend on the electrical conductivity values for the fracture or matrix. Indeed, for the simple case of a horizontal fracture with the prescribed boundary conditions, the resulting potential only depends upon the lateral coordinate $x$. Also note that, for this configuration, the discrete inverse Fourier-cosine transform described in Appendix A cannot be used since the corresponding optimized coefficients are defined for configurations with pointsource injections. Thus we conduct our validation in the Fourier domain considering equation (22) as our reference solution.

Figure 3 shows the Fourier-cosine transform of the electric potential $\bar{\phi}_{S C}$ computed with the reference analytical solution (22) and with our DDP approach. For the latter, the potential obtained in both the fracture and the matrix is shown. These results were determined for a domain of length $L=1 \mathrm{~m}$, which was discretized into 101 blocks in each direction. The fracture aperture $b_{f}$ was set to $10^{-3} \mathrm{~m}$ and the electrical conductivities $\sigma_{f}$ and $\sigma_{m}$ were set to $10^{-1} \mathrm{~S} / \mathrm{m}$ and $10^{-3} \mathrm{~S} / \mathrm{m}$, respectively. We see good agreement in the figure between our numerical approach and the analytical solution, as well as different behaviors of $\bar{\phi}_{S C}$ depending on the considered value of $\omega$. For small values of $\omega$, $\bar{\phi}_{S C}$ decreases linearly as $x$ increases (Figure 3a-b), and for large values of $\omega, \bar{\phi}_{S C}$ either increases (Figure 3c) or decreases (Figure 3d) until it reaches a constant value. Note that the same results were obtained for different ratios of the electrical conductivities $\sigma_{f}$ and $\sigma_{m}$ and for a larger number of horizontal fractures (results not shown).

\subsubsection{Multiple-fracture configurations}

In order to investigate more complex configurations involving multiple fractures, we now consider the three $30 \times 30 \mathrm{~m}$ fractured domains presented in Figure 4 . The matrix and fracture electrical conductivities for all examples were set to $\sigma_{m}=10^{-5} \mathrm{~S} / \mathrm{m}$ and $\sigma_{f}=10^{-2} \mathrm{~S} / \mathrm{m}$, respectively. Note that these configurations are considered in the present section to validate our 2.5D modeling approach 


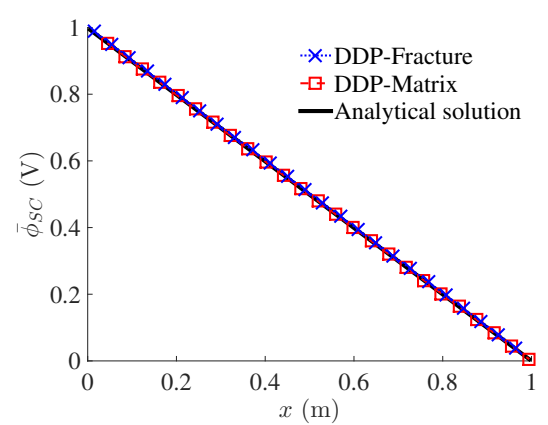

(a)

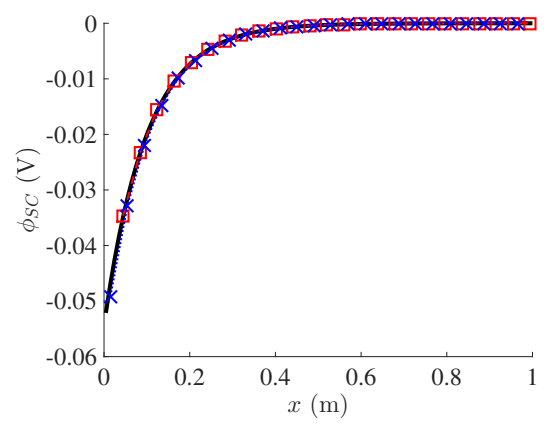

(c)

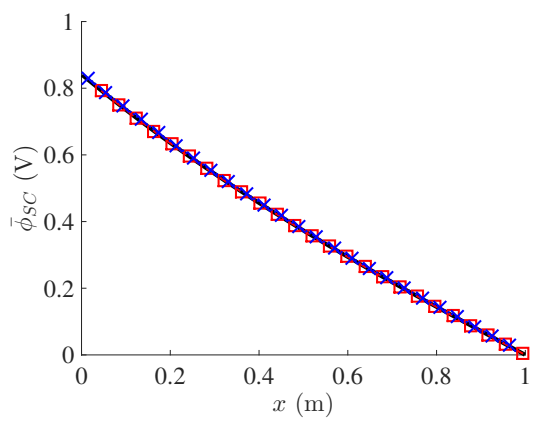

(b)

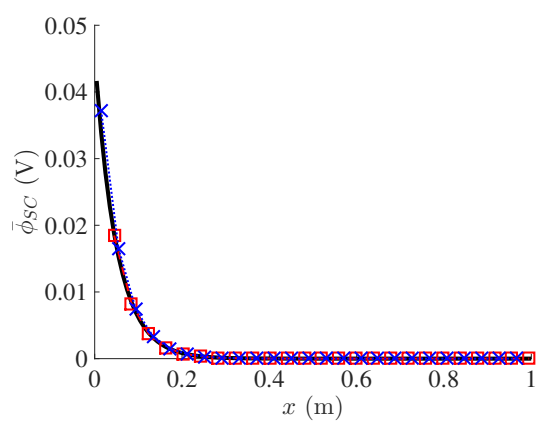

(d)

Figure 3: Fourier-cosine transform of the electric potential (in V) for the case of a single horizontal fracture as a function of the lateral coordinate $x$. Results were obtained using our DDP approach (both in the matrix and in the fracture) and using the analytical solution (22). The wavenumber $\omega$ was set to (a) 0.1 , (b) 1, (c) 10, and (d) 20 .

whereas in Section 4 they are used to simulate electrical resistivity measurements in fractured rocks.

For the validation, we assume the same type of point-source injections and boundary conditions that were considered previously; that is, $1 \mathrm{~A}$ and $-1 \mathrm{~A}$ surface current injections located at $x=10 \mathrm{~m}$ and $x=20 \mathrm{~m}$, respectively, an insulating condition along the top boundary, and mixed boundary conditions along the sides and bottom.

For each configuration in Figure 4, we would like to validate by comparing the electric potential distribution obtained using our 2.5D modeling approach with that computed using the COMSOL Multiphysics 4.3 finite-element software package, the latter of which serves as the reference solution. Unfortunately, we found that these multiple-fracture examples led to prohibitive computational costs with COMSOL when all of the fractures were considered and a realistic fracture aperture of $b=10^{-3} \mathrm{~m}$ was used. Indeed, both meshing and solving the corresponding finite-element system were found to overwhelm available computational resources because of the small scale of the fractures compared to the domain size. For this reason, we simplified the considered domains in Figure 4 for our validation as follows: (i) the fracture aperture was set to $10^{-2} \mathrm{~m}$, instead of $10^{-3} \mathrm{~m}$, in order to facilitate the 


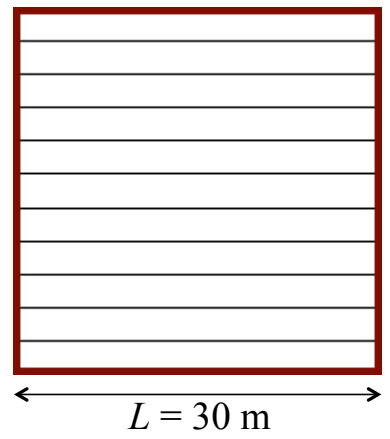

(a) DFN1

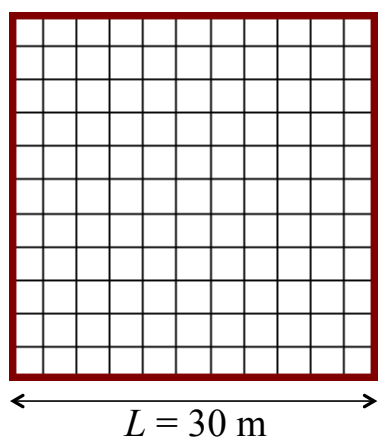

(b) DFN2

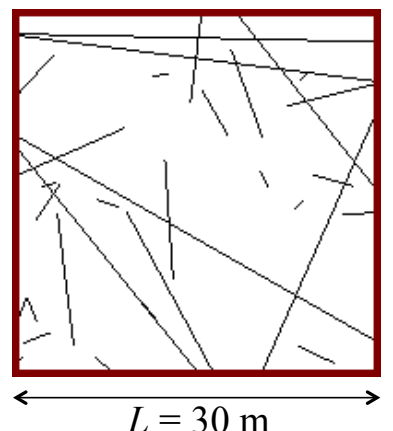

(c) DFN3

Figure 4: Multiple-fracture configurations used to validate our numerical approach.

meshing inside each fracture; (ii) the fractures located below $5 \mathrm{~m}$ depth were removed as these fractures will have minimal impact on the surface measurements for the studied experiment; (iii) the fractures that do not connect the borders of the domain were removed; and (iv) for DFN2, all of the vertical fractures were removed except for the two closest to each point source. Note that these simplifications were made only for our validation in order to reduce the numerical cost of the COMSOL simulations while keeping the most important fractures of the system.

Figures 5a-c show the simplified fractured domains as well as the corresponding distribution of the 
electric potential perturbation $\phi^{*}$, related to the presence of the fractures, which is defined as

$$
\phi^{*}(x, z)=\left|\phi\left(x, y_{0}, z\right)-\phi_{m}\left(x, y_{0}, z\right)\right|,
$$

where $\phi\left(x, y_{0}, z\right)$ is the electric potential of the fractured porous domain, and $\phi_{m}\left(x, y_{0}, z\right)$ is the electric potential corresponding to an unfractured porous domain having constant electrical conductivity $\sigma_{m}$. The distribution of $\phi^{*}$ was evaluated using our 2.5D modeling approach using 200 matrix blocks in each direction, which led to roughly $4 \times 10^{4}$ meshing elements and a total computational time of approximately 3 minutes for each fractured domain. In comparison, the number of meshing elements required by COMSOL was more than $10^{6}$ in each case, and the total computational time was roughly 3 times greater for DFN1 and DFN2 and 65 times greater for DFN3. Also plotted in Figure 5d is the electric potential perturbation at the surface $\phi_{S}^{*}=\phi^{*}(x, 0)$ computed using our code and COMSOL. Here we see an excellent agreement between the two codes, which confirms the validation of our modeling approach for the multiple fractures case.

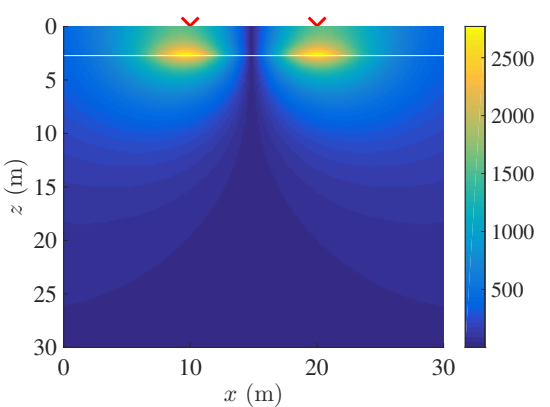

(a)

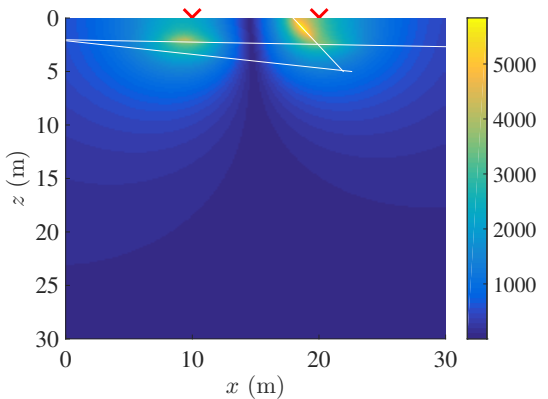

(c)

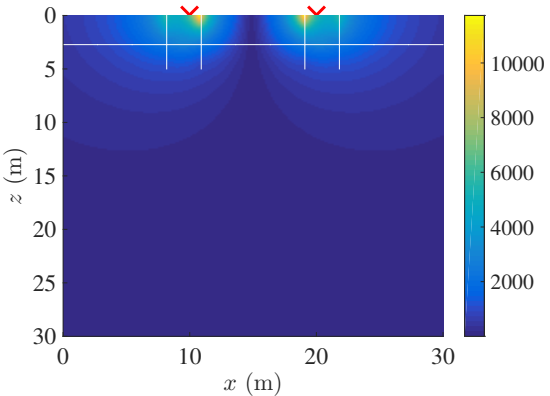

(b)

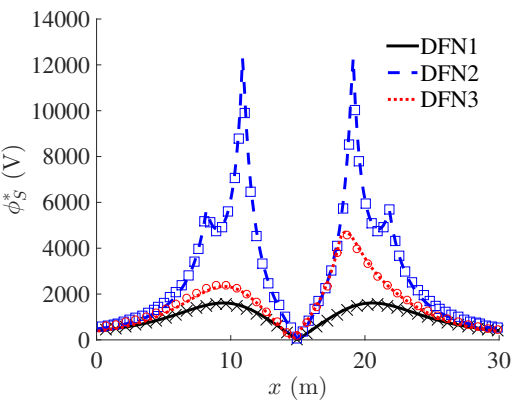

(d)

Figure 5: (a-c) Electric potential perturbation $\phi^{*}$ (in V) obtained using our 2.5D DDP approach after simplification of the fractured domains (a) DFN1, (b) DFN2, and (c) DFN3 from Figure 4. The white lines in (a-c) represent the fractures and the red symbols show the locations of the current electrodes. (d) Electric potential perturbation at the surface, $\phi_{s}^{*}$ (in $\mathrm{V}$ ), plotted as a function of $x$ and obtained using our approach (symbols) and the COMSOL finite-element solution (lines). 


\section{Results}

We now compute using our 2.5D modeling approach a variety of four-electrode resistivity measurements on the fractured domains shown in Figure 4, in order to simulate the type of data that would be acquired during a typical tomographic geoelectrical survey. To this end, we consider the three Wenner electrode configurations presented in Figure 6, each of which corresponds to a different electrode spacing $s$, which are progressively moved along the Earth's surface by an amount equal to the unit spacing between the electrodes $u=0.9 \mathrm{~m}$. Current injections of $1 \mathrm{~A}$ and $-1 \mathrm{~A}$ are performed at A and B, respectively, and we consider the same boundary conditions that were used for the validation (Section 3.2.2). Now, however, the fracture aperture is prescribed a more realistic value of $10^{-3} \mathrm{~m}$. Using our 2.5D DDP modeling approach with 100 matrix blocks in each direction, we compute the absolute difference in potential between $\mathrm{M}$ and $\mathrm{N}$, denoted as $V_{M N}$.

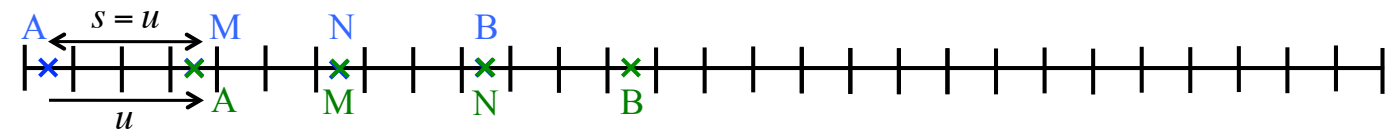

(a) W1

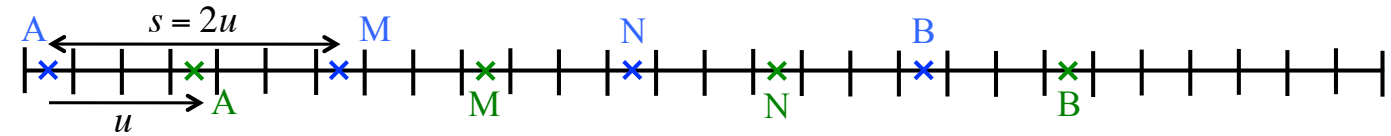

(b) W2

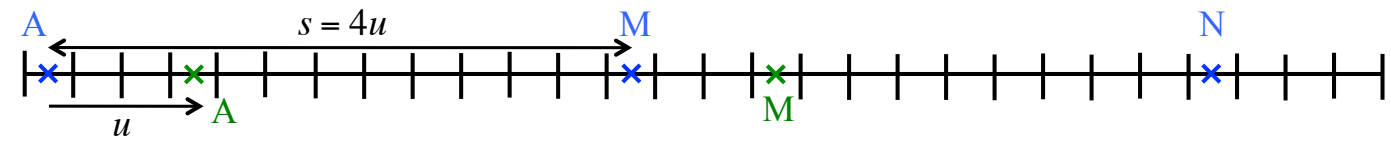

(c) W4

Figure 6: Considered Wenner electrode configurations where the electrode spacing $s$ is set equal to (a) $u$ (W1), (b) $2 u$ (W2), and (c) $4 u$ (W4). The small vertical lines represent the domain discretization. The electrode translation was set to $u=0.9 \mathrm{~m}$ for all experiments, and the electrodes in blue and green correspond to the first and second measurements, respectively.

From the absolute difference in potential $V_{M N}$, we calculate the apparent electrical resistivity $\rho_{a}=$ $2 \pi s V_{M N}$ (e.g., Telford et al., 1990). For a homogeneous porous domain having electrical conductivity $\sigma_{m}$, we found the apparent electrical resistivity $\rho_{a}^{m}$ to well approximate $1 / \sigma_{m}$ with an error smaller than $4 \%$ for each electrode configuration. We consider this small level of error to be acceptable because it is expected that some inaccuracies will arise from the discretization as well as from the numerical Fourier inversion. However, as this error depends on the considered electrode configuration and as we aim to compare the results obtained for different configurations, we define the normalized apparent 
resistivity $\rho_{a}^{*}=\rho_{a} \times \rho^{m} / \rho_{a}^{m}$ with $\rho^{m}=10^{5} \Omega \cdot \mathrm{m}$. Figure 7 shows $\rho_{a}^{*}$ calculated as a function of the lateral position of the center of the electrode array $x_{M N}$, for the three fracture configurations shown in Figure 4 and the three Wenner spacings shown in Figure 6.

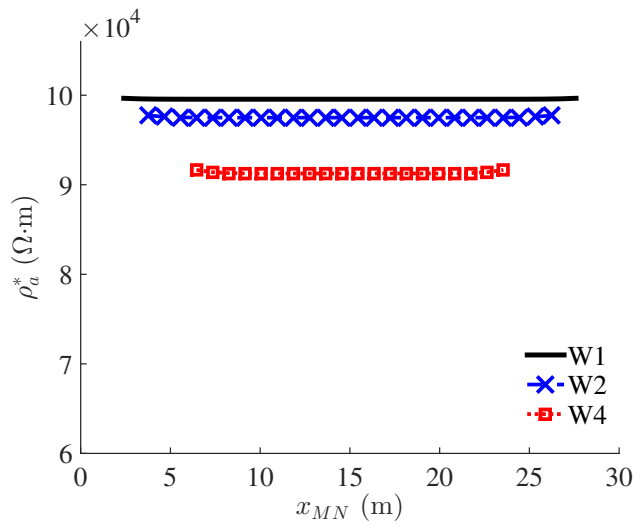

(a) DFN1

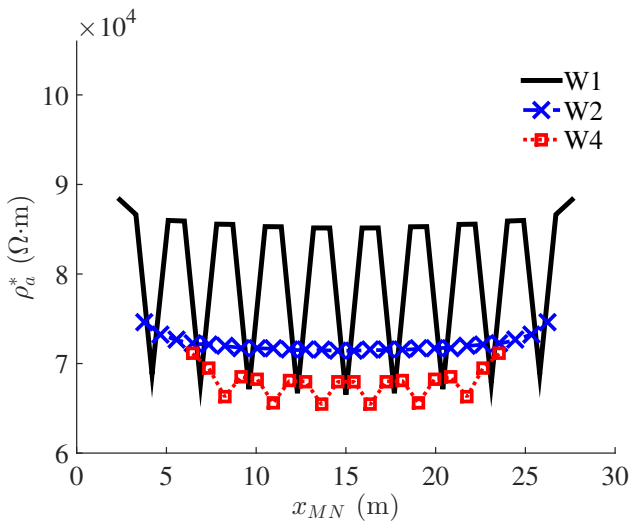

(b) DFN2

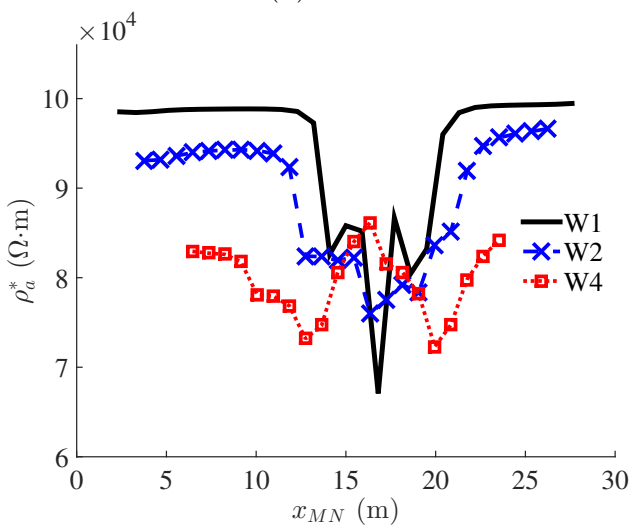

(c) DFN3

Figure 7: Normalized apparent resistivity $\rho_{a}^{*}$, plotted as a function of the lateral position of the center of the electrode array $x_{M N}$, for the fractured porous domains (a) DFN1, (b) DFN2, and (c) DFN3 from Figure 4 and the experiments W1, W2, and W4 presented in Figure 6.

For the fractured domains, we wish to determine which fractures impact the normalized apparent 
resistivity $\rho_{a}^{*}$. To this end, we define $\rho_{a}^{*}(d)$ as the resistivity evaluated by taking into account only the fractures located above depth $d$. With this definition, the results presented in Figure 7 correspond to $\rho_{a}^{*}(L)$ with $L$ equal to the total depth of the domain (i.e., taking into account all of the fractures). Considering $\rho_{a}^{*}(L)$ as a reference value, we define the depth of influence $d^{*}$ of the fractures as the smallest depth for which the average relative error in resistivity is smaller than $1 \%$. The latter value was chosen to provide close agreement between $\rho_{a}^{*}(d)$ and $\rho_{a}^{*}(L)$, such that $d^{*}$ represents the depth above which fractures significantly impact the behavior of $\rho_{a}^{*}$. The values of $d^{*}$ calculated for each fractured domain and electrode configuration in Figures 4 and 6 are presented in Table 1, and the corresponding equivalent fractured domains (i.e., ignoring fractures below depth $d^{*}$ ) are shown in Figure 8. For comparison, note that the approximate depth of influence of a homogeneous half space is defined as half of the electrode spacing (e.g., Binley \& Kemna, 2005), which leads to a depth of investigation equal to $0.45 \mathrm{~m}, 0.9 \mathrm{~m}$, and $1.8 \mathrm{~m}$ in experiments $\mathrm{W} 1, \mathrm{~W} 2$, and $\mathrm{W} 4$, respectively.

\begin{tabular}{c|c|c|c} 
& DFN1 & DFN2 & DFN3 \\
\hline W1 & 0 & 2.8 & 2.2 \\
\hline W2 & 2.8 & 4.3 & 2.9 \\
\hline W4 & 5.5 & 8.2 & 5.2
\end{tabular}

Table 1: Values of the depth of influence of the fractures $d^{*}$ (in $\mathrm{m}$ ) for the domains in Figure 4 and the electrode configurations in Figure 6. These values were determined up to a precision of $0.1 \mathrm{~m}$.

For the parallel fracture case (DFN1), we see that $\rho_{a}^{*}$ is constant as a function of position $x_{M N}$ for all experiments (Figure 7a). In addition, we observe that this constant value is (i) equal to the apparent resistivity for the corresponding unfractured porous domain, $\rho^{m}=10^{5} \Omega \cdot \mathrm{m}$, in experiment W1; (ii) smaller than $\rho^{m}$ in experiments $\mathrm{W} 2$ and W4; and (iii) smaller for experiment W4 than for experiment W2. As shown in Table 1, this behavior results from an increase in $d^{*}$ with an increase of the electrode spacing $s$. More precisely, when $s$ is equal to $u$ (W1), the fractures do not impact the value of $\rho_{a}^{*}$ (Figure 8a). Increasing $s$ from $u$ (W1) to $2 u$ (W2) means that the top fracture of the domain impacts $\rho_{a}^{*}$ (Figure 8d), and increasing $s$ from $2 u$ (W2) to $4 u$ (W4) means that the top two fractures impact $\rho_{a}^{*}$ (Figure $8 \mathrm{~g}$ ).

For the case of horizontal and vertical fractures (DFN2), oscillations of $\rho_{a}^{*}$ are observed with experiments W1 and W4 (Figure 7b). These oscillations correspond to successions of configurations where a different number of fractures is present between the current electrodes. For W1, the largest and smallest values of $\rho_{a}^{*}$ occur when one and two fractures, respectively, are located between electrodes $\mathrm{A}$ and $\mathrm{B}$, and for $\mathrm{W} 4$, the largest and smallest values occur when four and five fractures, respectively, are located between these electrodes. Although successions of configurations with different numbers 


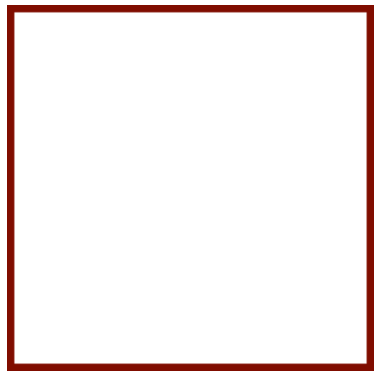

(a) DFN1, W1

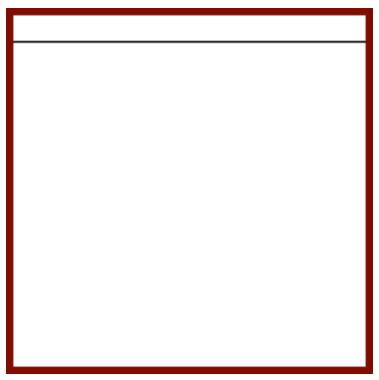

(d) DFN1, W2

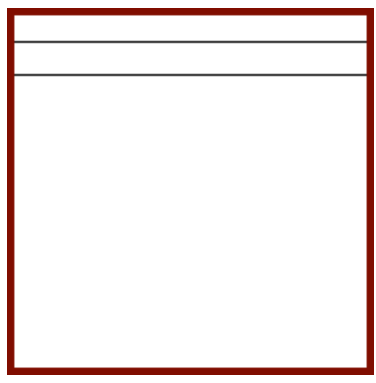

(g) DFN1, W4

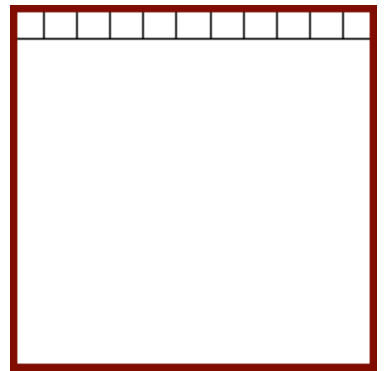

(b) DFN2, W1

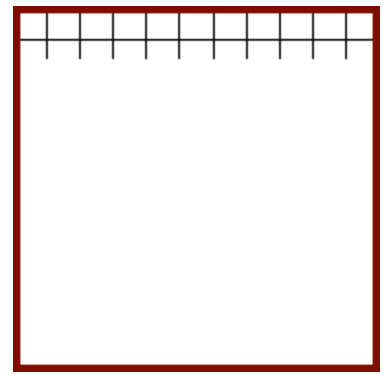

(e) DFN2, W2

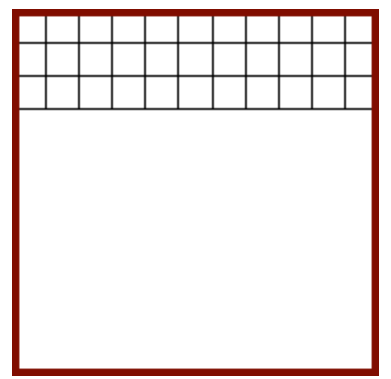

(h) DFN2, W4

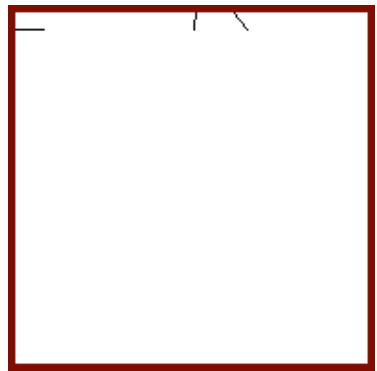

(c) DFN3, W1

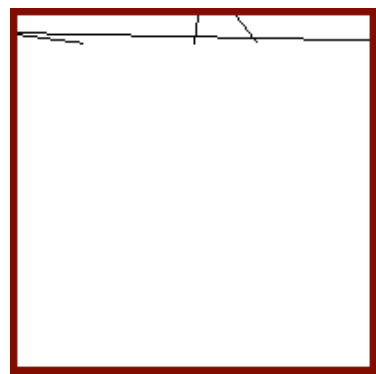

(f) DFN3, W2

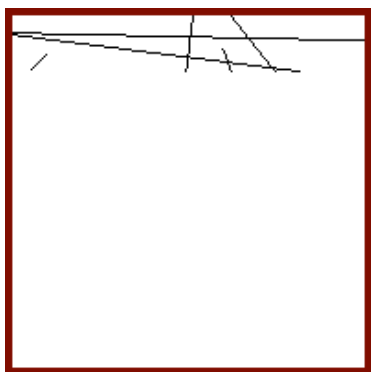

(i) DFN3, W4

Figure 8: Equivalent domains corresponding to the fracture configurations DFN1 (first column), DFN2 (second column), and DFN3 (third column) from Figure 4, and for the Wenner electrode configurations W1 (first row), W2 (second row), and W4 (third row) from Figure 6.

of fractures between the current electrodes also occur in W2, oscillations of $\rho_{a}^{*}$ are not observed. We believe that this behaviour is related to different configurations of the vertical fractures at the depths of influence. In W1 and W4, the lower extremities of these fractures reach a horizontal fracture (Figure 8b and $\mathrm{h}$ ), whereas in W2 these extremities are embedded in the rock matrix (Figure 8e). Note that, as before and as could be expected, increasing $s$ results in increasing $d^{*}$ (Table 1) and thus the number of fractures impacting the value of $\rho_{a}^{*}$ (Figure $8 \mathrm{~b}$, e, and $\mathrm{h}$ ).

Finally, for the random fracture case (DFN3) considering electrode configuration W1 (Figure 7c), we observe that (i) $\rho_{a}^{*}$ is slightly smaller than $\rho^{m}$ when $x_{M N}$ is less than $12.3 \mathrm{~m}$; (ii) $\rho_{a}^{*}$ presents large variations and reaches its smallest values when $x_{M N}$ is between $12.3 \mathrm{~m}$ and $21.3 \mathrm{~m}$; and (iii) 
$\rho_{a}^{*}$ is close to $\rho^{m}$ when $x_{M N}$ is larger than $21.3 \mathrm{~m}$. Studying the fractures present above the depth of influence $d^{*}$ (Figure 8c) shows that these observations result, respectively, from (i) the presence of a small horizontal fracture in the top-left corner of the domain; (ii) the presence of two sub-vertical fractures at the top of the domain near the center; and (iii) the absence of fractures in the top-right corner of the domain. In comparison with W1, conducting experiment W2 results in (i) a decrease of the maximum value of $\rho_{a}^{*}$ in that it is now always smaller than $\rho^{m}$; (ii) smaller values of $\rho_{a}^{*}$ on the left-hand side, here for $x_{M N} \leq 10.95 \mathrm{~m}$, than on the right-hand side of the domain, here for $x_{M N} \geq 22.65 \mathrm{~m}$; and (iii) a wider extent of the area where the smallest values of $\rho_{a}^{*}$ are observed, here for $x_{M N}$ from $10.95 \mathrm{~m}$ to $22.65 \mathrm{~m}$. Figure 8f shows that these observations can be explained, respectively, by: (i) the presence of a sub-horizontal fracture extending across the entire domain; (ii) the presence of another short sub-horizontal fracture near the top-left corner of the domain; and (iii) the larger extent, in comparison with W1, of the sub-vertical fractures. Finally, the results obtained with configuration W4 show (i) a decrease in the largest values of $\rho_{a}^{*}$ in comparison with W1 and W2; (ii) smaller values of $\rho_{a}^{*}$ on the left side, for $x_{M N} \leq 9.15 \mathrm{~m}$, than on the right side of the domain, for $x_{M N} \geq 23.55 \mathrm{~m}$; and (iii) two regions with a strong decrease and increase of $\rho_{a}^{*}$. These results are explained by the presence of an additional sub-horizontal fracture using configuration W4 (Figure 8i), in comparison with W2 (Figure 8f), which implies that the largest values of $\rho_{a}^{*}$ are smaller in the former than in the latter configuration. As this additional fracture does not reach the right-hand side of the domain, it also implies that larger values of $\rho_{a}^{*}$ are observed on this side than on the left-hand side with configuration W4. In addition, the two sub-vertical fractures have different characteristics between the W2 (Figure 8f) and W4 (Figure 8i) configurations, as the distance between the bottom extremities of these fractures is larger in the latter case than in the former case. This implies that they are separated enough using W4 to individually impact $\rho_{a}^{*}$ and produce two distinct decreases in $\rho_{a}^{*}$.

\section{Conclusions}

We have presented in this paper a $2.5 \mathrm{D}$ discrete-dual-porosity approach for numerically modeling electric current flow in fractured media. To our knowledge, this is the first attempt to develop a computationally efficient algorithm that (i) is well adapted to the numerical challenges arising from the specificities of fractured rocks, and (ii) adequately represents the physics of point-source injections in heterogeneous domains. We have validated our approach for both unfractured and fractured porous domains using a variety of fracture networks. Comparison with a standard finite-element solution for 
cases involving multiple fractures clearly demonstrates the numerical efficiency of our approach.

The results presented in this work indicate that a small number of millimeter-scale fractures can significantly impact the apparent electrical resistivity evaluated from ERT surveys. For example, the presence of only two horizontal fractures having aperture $10^{-3} \mathrm{~m}$ and electrical conductivity three orders of magnitude larger than the surrounding matrix results in a decrease in $10 \%$ of the apparent electrical resistivity. As expected, this impact depends on the considered electrode configurations; increasing the electrode spacing, for example, results in an increase in the number of fractures impacting the measured resistivity. Our results also show that the presence of horizontal fractures extending from the left to right sides of the considered domains results in a decrease of the measured resistivity everywhere along the electrode line. Conversely, the presence of vertical fractures results in localized decreases in this resistivity. In the latter case, it is important to note that the vertical fractures may not be situated where the decreases in resistivity are observed, as (i) the changes in resistivity can result from variations in the number of fractures between the current electrodes; and (ii) the vertical fractures need to be separated enough to individually impact the apparent resistivity.

Our results open new perspectives in terms of the inversion of geoelectrical data in order to characterize fractured rocks. In particular, we question to what extent such data may be used to progressively reconstruct the properties of the underlying fracture network, either deterministically or stochastically. In this regard, future work will include statistical investigation of the results obtained for random fracture networks with large ranges in their geometrical properties. Finally, we wish to extend the work presented in this paper to "real" three-dimensional fractured-rock configurations, where reliance upon a $2.5 \mathrm{D}$ representation is not necessary. To this end, we are currently developing a $3 \mathrm{D}$ formulation of the discrete-dual-porosity modeling approach with special efforts to reduce the computational cost. This new modeling tool will enable us to simulate azimuthal resistivity surveys in fractured porous media in order to study (i) how these experiments help to identify the presence of fractures and evaluate their properties; and (ii) how the corresponding results might be integrated into an inversion framework.

\section{A Appendix A: Discrete inverse Fourier-cosine transform}

Consider that the space domain is discretized into $N_{y}$ elements of constant length $\Delta y$ in the $y$-direction. The resulting discretized values are defined as $y_{m}=(m-1 / 2) \Delta y$ with $m=1, \ldots, N_{y}$ and the electric potential $\phi$ at position $y_{m}$ is denoted as $\phi_{m}=\phi\left(x, y_{m}, z\right)$. Using a discretized formulation of the 
inverse Fourier-cosine transform (3b), the electric potential $\phi_{m}$ can be expressed as

$$
\phi_{m}=\frac{2}{\pi} \sum_{n=1}^{N_{w}} \bar{\phi}_{n} \cos \left(\omega_{n} y_{m}\right) \Delta \omega .
$$

In expression (24), the wavenumber $\omega$ is discretized into $N_{\omega}$ values of constant difference $\Delta \omega$ which are defined as $\omega_{n}=n \Delta \omega$ with $n=1, \ldots, N_{\omega}$. We set the discretization steps $\Delta \omega$ and $\Delta y$ to $\pi / T$ and $T / N_{y}$, respectively, with $T=100$ in our study. Assuming $N_{w}=N_{y}$ and considering $N$ such as $N=N_{w}=N_{y}$, expression (24) becomes

$$
\phi_{m}=\frac{2}{T} \sum_{n=1}^{N} \bar{\phi}_{n} \cos \left[\frac{n(2 m-1) \pi}{2 N}\right] \text {, }
$$

and can be written as

$$
\phi_{m}=\sum_{n=1}^{N} \bar{\phi}_{n} g_{n}
$$

where the coefficients $g_{n}$ are the Fourier weights.

To obtain an accurate evaluation of $\phi_{m}$ from expression (25), a fine discretization might be required, which will result in a large number of wavenumber $N_{\omega}$. As this number corresponds to the number of times that equation (2) has to be solved, a large value of $N_{\omega}$ results in a high computational cost. To reduce this cost, $\mathrm{Xu}$ et al. (2000) optimized the selection of the wavenumber and Fourier-weight values. Considering a point-source injection in homogeneous and heterogeneous half-space domains, they determine the following values for the wavenumber $\omega_{n}$ and Fourier weight $g_{n}$ :

$$
\omega_{1}=0.0217102 \quad \omega_{2}=0.2161121 \quad \omega_{3}=1.0608400 \quad \omega_{4}=5.0765870
$$

$$
g_{1}=0.0463660 \quad g_{2}=0.2365931 \quad g_{3}=1.0382080 \quad g_{4}=5.3648010
$$

These coefficients are used in our study for inverting the analytical and numerical results which are obtained in the Fourier domain. 


\section{B Appendix B: Singularity removal technique}

Considering point-source injections results in the presence of singularities at the locations of these injections where a large error in the electric potential can be observed. Although this error could be reduced by using a finer spatial discretization close to the singularities, a correction of these singularities is usually preferred in order to reduce the related numerical cost. Techniques to remove the source singularity have been developed for finite-difference and finite-element approaches (e.g., Li \& Spitzer, 2002; Lowry et al., 1989) by expressing the electric potential $\bar{\phi}$ in the Fourier domain as

$$
\bar{\phi}=\bar{\phi}^{r}+\bar{\phi}^{s}
$$

with $\bar{\phi}^{r}$ and $\bar{\phi}^{s}$ the regular and singular parts of the potential, respectively. Defining the latter potential as the Fourier transform of the electric potential in a semi-infinite half-space of constant electrical conductivity $\sigma_{0}, \bar{\phi}^{s}$ is expressed as

$$
\bar{\phi}^{s}=\frac{I}{2 \pi \sigma_{0}} \mathcal{K}_{0}\left(\omega \sqrt{\left(x-x_{0}\right)^{2}+z^{2}}\right) .
$$

From its definition, $\bar{\phi}^{s}$ is solution for equation (2) with $\sigma(x, z)=\sigma_{0}$, and $\sigma_{0}$ is defined as either the average conductivity over the whole domain (e.g., Lowry et al., 1989) or the conductivity at the pointsource location (e.g., Zhao \& Yedlin, 1996). As $\bar{\phi}$ is also solution for equation (2), $\bar{\phi}^{r}$ is solution for the following equation:

with $\sigma^{*}(x, z)=\sigma(x, z)-\sigma_{0}$.

Here, we wish to adapt the existing techniques to remove singularities in the modified finite-volume approach presented in Section 2.2.3. Note that the considered method will also be applicable to standard finite volume approaches. After integrating equation (30) over each matrix block volume $V_{I, J}$, we observe that the left- and right-hand sides of this equation have a similar formulation to the left hand-side of equation (12), implying that the same discretization technique can be used. This 
results in the following discretized expression

$$
\begin{aligned}
& C_{I, J} \bar{\phi}_{I, J}^{r}+C_{I, J}^{W} \bar{\phi}_{I-1, J}^{r}+C_{I, J}^{E} \bar{\phi}_{I+1, J}^{r}+C_{I, J}^{S} \bar{\phi}_{I, J-1}^{r}+C_{I, J}^{N} \bar{\phi}_{I, J+1}^{r} \\
& =C_{I, J}^{*} \bar{\phi}_{I, J}^{s}+C_{I, J}^{*, W} \bar{\phi}_{I-1, J}^{s}+C_{I, J}^{*, E} \bar{\phi}_{I+1, J}^{s}+C_{I, J}^{*, S} \bar{\phi}_{I, J-1}^{s}+C_{I, J}^{*, N} \bar{\phi}_{I, J+1}^{s},
\end{aligned}
$$

where the coefficients $C_{I, J}, C_{I, J}^{W}, C_{I, J}^{E}, C_{I, J}^{S}$, and $C_{I, J}^{N}$ are given in (14) and the coefficients $C_{I, J}^{*}$ and $C_{I, J}^{*, K}$ are defined as $C_{I, J}^{*}=C_{I, J}^{0}-C_{I, J}$ and $C_{I, J}^{*, K}=C_{I, J}^{0, K}-C_{I, J}^{K}(K=W, E, S, N)$ with $C_{I, J}^{0}$ and $C_{I, J}^{0, K}$ the counterparts of the coefficients $C_{I, J}$ and $C_{I, J}^{K}$ considering the constant electrical conductivity $\sigma_{0}$.

\section{Appendix C: Mixed boundary conditions}

When simulating ERT experiments, mixed boundary conditions are very often applied to the left, right and bottom borders of the considered domains (e.g., Dey \& Morrison, 1979; Li \& Spitzer, 2002; Rücker et al., 2006). These conditions help to reproduce the natural behavior of the electric potential at positions far away from the point-source injection. This implies that the size of the computational domain and the related computational cost can be reduced in comparison with other boundary conditions that might affect the observed results. Mixed boundary conditions in the Fourier domain are defined as

$$
\alpha(x, z) \bar{\phi}+\beta(x, z) \frac{\partial \bar{\phi}}{\partial \vec{n}}=\gamma(x, z)
$$

where $\vec{n}$ is the outward normal on which the boundary conditions are applied and position $(x, z)$ is located on one of the domain borders. As done in Dey \& Morrison (1979), we set the coefficients $\beta$ and $\gamma$ to 1 and 0 , respectively, and we define $\alpha$ as

$$
\alpha=\omega \frac{\mathcal{K}_{1}(\omega r)}{\mathcal{K}_{0}(\omega r)}\left(\frac{\vec{n} \cdot \vec{r}}{r}\right)
$$

where $\mathcal{K}_{1}$ is the modified Bessel function of the second kind of order $1, \omega$ is the wavenumber associated with the space-variable $y$, and $r=\sqrt{\left(x-x_{0}\right)^{2}+z^{2}}$ is the distance from the considered position $(x, z)$ on the domain border to the source point located at position $\left(x_{0}, z_{0}\right)$ with $z_{0}=0$.

To apply these boundary conditions in our DDP approach, we consider a ghost-cell method which leads to

$$
-\frac{\partial \bar{\phi}}{\partial \vec{n}}=\frac{2 \alpha}{2+\alpha \Delta} \bar{\phi}
$$


with $\Delta$ the cell size and $\bar{\phi}$ the cosine-Fourier transform of the electric potential at the boundary condition location.

\section{References}

Bateman, H., 1954. Bateman Manuscript Project: Tables of Integral Transforms, McGraw-Hill.

Berkowitz, B., 2002. Characterizing flow and transport in fractured geological media: A review, Advances in Water Resources, 25(8-12), 861-884.

Binley, A. \& Kemna, A., 2005. DC Resistivity and Induced Polarization Methods, in Hydrogeophysics, vol. 50 of Water Science and Technology Library, pp. 129-156, eds Rubin, Y. \& Hubbard, S. S., Springer Netherlands.

Boadu, F., Gyamfi, J., \& Owusu, E., 2005. Determining subsurface fracture characteristics from azimuthal resistivity surveys: A case study at Nsawam, Ghana, Geophysics, 70(5), B35-B42.

Bonnet, E., Bour, O., Odling, N. E., Davy, P., Main, I., Cowie, P., \& Berkowitz, B., 2001. Scaling of fracture systems in geological media, Reviews of Geophysics, 39(3), 347-383.

Brown, S. R., 1989. Transport of fluid and electric current through a single fracture, Journal of Geophysical Research: Solid Earth, 94(B7), 9429-9438.

Busby, J., 2000. The effectiveness of azimuthal apparent-resistivity measurements as a method for determining fracture strike orientations, Geophysical Prospecting, 48(4), 677-695.

Carneiro, J. F., 2009. Numerical simulations on the influence of matrix diffusion to carbon sequestration in double porosity fissured aquifers, International Journal of Greenhouse Gas Control, 3(4), 431-443.

Dershowitz, W. \& Miller, I., 1995. Dual porosity fracture flow and transport, Geophysical Research Letters, 22(11), 1441-1444.

Dey, A. \& Morrison, H., 1979. Resistivity modeling for arbitrarily shaped two-dimensional structures, Geophysical Prospecting, 27(1), 106-136.

Dorn, C., Linde, N., Le Borgne, T., Bour, O., \& Baron, L., 2011. Single-hole GPR reflection imaging of solute transport in a granitic aquifer, Geophysical Research Letters, 38.

Gautam, P. \& Mohanty, K., 2004. Matrix-fracture transfer through countercurrent imbibition in presence of fracture fluid flow, Transport in Porous Media, 55(3), 309-337. 
Greenhalgh, S., Marescot, L., Zhou, B., Greenhalgh, M., \& Wiese, T., 2009a. Electric potential and fréchet derivatives for a uniform anisotropic medium with a tilted axis of symmetry, Pure and applied geophysics, 166(4), 673-699.

Greenhalgh, S., Zhou, B., Greenhalgh, M., Marescot, L., \& Wiese, T., 2009b. Explicit expressions for the fréchet derivatives in 3d anisotropic resistivity inversion, Geophysics, 74(3), F31-F43.

Herwanger, J., Worthington, M., Lubbe, R., Binley, A., \& Khazanehdari, J., 2004a. A comparison of cross-hole electrical and seismic data in fractured rock, Geophysical prospecting, 52(2), 109-121.

Herwanger, J. V., Pain, C. C., Binley, A., De Oliveira, C. R. E., \& Worthington, M. H., 2004 b. Anisotropic resistivity tomography, Geophysical Journal International, 158(2), 409-425.

Kirkby, A., Heinson, G., \& Krieger, L., 2016. Relating permeability and electrical resistivity in fractures using random resistor network models, Journal of Geophysical Research: Solid Earth.

Lane, J., Haeni, F., \& Watson, W., 1995. Use of a square-array Direct-Current resistivity method to detect fractures in crystalline bedrock in New-Hampshire, Ground Water, 33(3), 476-485.

Li, Y. \& Spitzer, K., 2002. Three-dimensional DC resistivity forward modelling using finite elements in comparison with finite-difference solutions, Geophysical Journal International, 151(3), 924-934.

Li, Y. \& Spitzer, K., 2005. Finite element resistivity modelling for three-dimensional structures with arbitrary anisotropy, Physics of the Earth and Planetary Interiors, 150(1), 15-27.

Lofi, J., Pezard, P., Loggia, D., Garel, E., Gautier, S., Merry, C., \& Bondabou, K., 2012. Geological discontinuities, main flow path and chemical alteration in a marly hill prone to slope instability: Assessment from petrophysical measurements and borehole image analysis, Hydrological Processes, 26(14), doi:10.1002/hyp.7997.

Long, J. C. S., Remer, J. S., Wilson, C. R., \& Witherspoon, P. A., 1982. Porous media equivalents for networks of discontinuous fractures, Water Resources Research, 18(3), 645-658.

Lowry, T., Allen, M., \& Shive, P., 1989. Singularity removal - A refinement of resistivity modeling techniques, Geophysics, 54(6), 766-774.

Neuman, S., 2005. Trends, prospects and challenges in quantifying flow and transport through fractured rocks, Hydrogeology Journal, 13(1), 124-147. 
Nguyen, F., Kemna, A., Robert, T., \& Hermans, T., 2016. Data-driven selection of the minimumgradient support parameter in time-lapse focused electric imaging, Geophysics, 81(1), A1-A5.

Pidlisecky, A. \& Knight, R., 2008. FW2_5D: A MATLAB 2.5-D electrical resistivity modeling code, Computers $\&$ Geosciences, 34(12), 1645-1654.

Ritzi, R. W. \& Andolsek, R. H., 1992. Relation between anisotropic transmissivity and azimuthal resistivity surveys in shallow, fractured, carbonate flow systems, Ground Water, 30(5), 774-780.

Robinson, J., Johnson, T., \& Slater, L., 2013. Evaluation of known-boundary and resistivity constraints for improving cross-borehole DC electrical resistivity imaging of discrete fractures, Geophysics, 78(3), D115-D127.

Rotter, B. E., Barry, D. A., Gerhard, J. I., \& Small, J. S., 2008. Modeling U(VI) biomineralization in single- and dual- porosity porous media, Water Resources Research, 44(8).

Roubinet, D. \& Irving, J., 2014. Discrete-dual-porosity model for electric current flow in fractured rock, Journal of Geophysical Research-Solid Earth, 119(2), 767-786.

Roubinet, D., de Dreuzy, J.-R., \& Davy, P., 2010. Connectivity-consistent mapping method for 2-D discrete fracture networks, Water Resources Research, 46, W07532.

Roubinet, D., Linde, N., Jougnot, D., \& Irving, J., 2016. Streaming potential modeling in fractured rock: Insights into the identification of hydraulically active fractures, Geophysical Research Letters, 43(10), 4937-4944.

Rücker, C., Günther, T., \& Spitzer, K., 2006. Three-dimensional modelling and inversion of DC resistivity data incorporating topography - I. Modelling, Geophysical Journal International, 166, $495-505$.

Shen, J., Su, B., \& Guo, N., 2009. Anisotropic characteristics of electrical responses of fractured reservoir with multiple sets of fractures, Petroleum Science, 6(2), 127-138.

Telford, W. M., Geldart, L. P., \& Sheriff, R. E., 1990. Applied Geophysics, Monograph series, Cambridge University Press.

Wishart, D. N., Slater, L. D., \& Gates, A. E., 2008. Fracture anisotropy characterization in crystalline bedrock using field-scale azimuthal self potential gradient, Journal of Hydrology, 358(1), 35-45. 
608

609

Xu, S., Duan, B., \& Zhang, D., 2000. Selection of the wavenumbers k using an optimization method for the inverse Fourier transform in 2.5D electrical modelling, Geophysical Prospecting, 48(5), 789-796.

Zhao, S. \& Yedlin, M., 1996. Some refinements on the finite-difference method for 3-D dc resistivity modeling, Geophysics, 61(5), 1301-1307. 